\title{
Laboratory measurements of the far-infrared to millimeter dust opacity of amorphous $\mathrm{Mg} / \mathrm{Fe}$ silicates
}

\author{
Pierre Mohr* \\ Astrophysical Institute and University Observatory, Friedrich-Schiller-University Jena, \\ Schillergaesschen 3, 07745 Jena, Germany \\ E-mail: pierre.mohr@uni-jena.de

\section{Harald Mutschke} \\ Astrophysical Institute and University Observatory, Friedrich-Schiller-University Jena, \\ Schillergaesschen 3, 07745 Jena, Germany \\ E-mail: harald.mutschkeeuni-jena.de
}

\section{Frank Lewen}

Institute of Physics I, University of Cologne, Zuelpicher Strasse 77, 50937 Cologne, Germany

E-mail: lewen@ph1.uni-koeln.de

\begin{abstract}
Amorphous silicates are expected to be one of the major constituents of dust in the interstellar medium. Laboratory investigations of these compounds are therefore crucial for bettering the understanding of the nature of interstellar dust. For our measurements, we used synthetic ana$\log$ materials, produced by quenching of melts with well-defined chemical compositions. We performed transmission measurements on a variety of pyroxene-like amorphous silicates with various $\mathrm{Mg} / \mathrm{Fe}$-ratios. The wavelength-dependent opacities of these samples in the wavelength range between $50 \mu \mathrm{m}$ and $1.2 \mathrm{~mm}$ and in the temperature range between $300 \mathrm{~K}$ and $10 \mathrm{~K}$ have been investigated. We observed not only a temperature dependence of the opacities, but also a clear dependence on the iron content. The reason for this behavior lies in the microstructure of amorphous silicates, but is not yet completely explored.
\end{abstract}

The Life Cycle of Dust in the Universe: Observations, Theory, and Laboratory Experiments - LCDU 2013, 18-22 November 2013

Taipei, Taiwan

\footnotetext{
* Speaker.
} 


\section{Introduction}

The galactic energy balance is significantly influenced by interstellar dust. It absorbs radiation at ultraviolet and visible wavelengths and re-emits it in the infrared and mm-wave range. At least $30 \%$ of the total amount of energy emitted by stars as starlight is absorbed and re-emitted by dust grains in this manner [1]. The low temperature of grains in the interstellar medium leads thereby to emission at long wavelengths.

It has been known for several decades that silicates are a fundamental constituent of interstellar dust [2]. Since their first detection in the late 1960s, the prominent silicate features at $10 \mu \mathrm{m}$ and 18 $\mu \mathrm{m}$ wavelength have been probed at several surveys. They occur similarly everywhere in the ISM of the Milky Way, independent of the line of sight. Although the observed features differ slightly in their shapes and intensities, depending on the observed region, they are all smooth and very broad. This excludes the presence of a significant amount of crystalline silicates, which would cause sharp and narrow features.

The low temperature optical properties of astronomically relevant silicate dust species at FIR and sub-mm wavelengths have been investigated by several authors in recent years. These investigations dealt mainly with pure magnesium silicates [3, 4], in one case additionally with a pure iron silicate [5]. The influence of a gradual integration of iron has never been studied before. However, all studies revealed a clear temperature dependence of the opacities of silicate grains, particularly of amorphous phases. While focusing on those same phases, we expand the currently available dataset with regard to the influence of the incorporation of Fe atoms into the amorphous network. Our data will be useful for the interpretation of observed spectra from current projects like SOFIA and ALMA, and should contribute to an understanding of observations from the recent space missions Herschel and Spitzer.

\section{Sample preparation and measurement procedure}

We synthesized a series of $\mathrm{Mg} / \mathrm{Fe}$-glasses with pyroxene-like chemism $\left(\mathrm{Mg}_{x} \mathrm{Fe}_{1-x} \mathrm{SiO}_{3}\right)$. This has been done by quenching of melts using rotating copper rolls [6]. Mixtures of certain amounts of magnesium carbonate $\left(\mathrm{MgCO}_{3}\right)$, iron oxalate $\left(\mathrm{C}_{2} \mathrm{FeO}_{4} \cdot 2 \mathrm{H}_{2} \mathrm{O}\right)$, and silicon dioxide powder $\left(\mathrm{SiO}_{2}\right)$ served thereby as precursors for our melts. Since the actual water content of chemicals may change due to surface reactions once they are exposed to air humidity for some time, we frequently checked the water content of our ingredients. Thus, we were able to determine the required mass of precursor material for the different compositions of melts much more precisely and, as a consequence, regulate the chemism of the resulting glasses with a higher accuracy. The glasses were melted at temperatures above $1600^{\circ} \mathrm{C}$. The samples, which were placed in a platinum crucible, were removed from the hT-oven at the maximum temperature and immediately poured between two copper rolls, of which one is rotating, driven by an electric engine. The fast cooling preserves the disordered state of the melt and avoids crystallization processes. Unfortunately, it is not possible to completely avoid some early stages of crystallization. The phase content depends on the iron content of the melt. While iron-free $\mathrm{MgSiO}_{3}$ is almost totally amorphous, a higher iron content tends to result in increased crystallinity. To get appropriate samples it is therefore necessary to remove crystallized areas. The phases are discriminable based on different optically visible characteristics. We separted 
amorphous from crystalline phases using a binocular microscope. After removing the crystalline fraction, the remaining glassy fragments were ground to a fine powder. A very small percentage of this powder was embedded into a $\mathrm{KBr}$ pellet in order to verify its amorphousness by a MIR absorption measurement.

To measure in the far-infrared region, hot pressed polyethylene pellets with a diameter of 13 $\mathrm{mm}$ were used. For measurements in the spectral range between $50 \mu \mathrm{m}$ and $300 \mu \mathrm{m}$ we chose a sample to PE ratio of 1:16 with a sample mass of $10 \mathrm{mg}$. In order to prohibit scattering effects at these relatively short wavelengths we selected grains with a size of $1 \mu \mathrm{m}$ or less by sedimentation in acetone. For wavelengths above $300 \mu \mathrm{m}$ we applied pellets with a sample to PE ratio of 8:1 and either $400 \mathrm{mg}$ or 2,4 $\mathrm{g}$ (or more) of sample material. The massive pellets deliver the most accurate values at wavelengths around $1 \mathrm{~mm}$ but are not measurable below $\sim 500 \mu \mathrm{m}$ because of their high optical depth.

We could obtain glasses in the compositional range between $\mathrm{MgSiO}_{3}$ and $\mathrm{Mg}_{0,5} \mathrm{Fe}_{0,5} \mathrm{SiO}_{3}$. We used scanning electron microscopy to check the chemical homogeneity of the samples and electron microprobe analysis to determine their chemical compositions. It turned out that the homogeneity also depends on the iron content. While glasses with a Fe/Mg ratio of 1:3 or lower are absolutely homogeneous, the presence of a higher amount of iron leads to some kind of phase separation into regions with different elemental abundances ( $\pm 5 \%$ for $\mathrm{Si}, \mathrm{Fe}$; less for $\mathrm{Mg}$ ), spatially separated by a few tens of microns and with smooth transitions from one to another. The separation takes place within the amorphous network and is not accompanied by crystallization. The mean composition of all samples deviates by less than 2 mass- $\%$ per element from the respective index values. This deviation is also not exceeded for the homogeneous samples.

For our FIR absorption measurements we used an FTIR spectrometer BRUKER IFS 113v. A LHe continuous flow cryostat, equipped with polyethylene windows, was placed directly into the optical path. For the 1:16 pellets we used a pellet made from pure polyethylene for the reference measurements. This approach is not practicable for the 8:1 pellets because the refractive index of the mixture is completely different from that of pure polyethylene. For that reason, we conducted measurements of the empty beam path as reference and calculated the reflection loses of the samples using effective medium approximations. The spectra obtained from measurements of 8:1 pellets with a sample amount of $400 \mathrm{mg}$ at wavelengths above $250 \mu \mathrm{m}$ show several fringes at periodic intervals. These fringes are caused by multiple reflections between the surfaces of the pellet. In order to remove these effects we did a least squares fit to the spectrum using a fifth-order polynomial. For all the other measured pellet concentrations and thicknesses interferences caused by reflections do not play an important role.

To adapt our measured absorptivities to those of free-flying single grains, we applied the Bruggeman model to compute theoretical mass absorption coefficients for single grains and agglomerates in a matrix. Thus we could calculate a correction factor, which we used to adapt our data derived from high-concentration pellet measurements. For sample concentrations $<1 / 3$ of the total volume, there is no way to compute theoretical absorptivities without assuming a certain grain shape. Therefore, the low-concentration pellet spectra were fitted to those obtained from highconcentration pellets. For a detailed description of the Bruggeman model and its application, we refer to papers by Henning et al. (1995) [7] and Boudet et al. (2005) [4]. 


\section{Results}

The wavelength dependent mass absorption coefficient $\kappa$ of $\mathrm{MgSiO}_{3}$ glass for temperatures between room temperature and $10 \mathrm{~K}$ is shown in Figure 1. To cover the indicated spectral range with the highest possible accuracy, spectra taken from measurements of three pellets have been merged for each temperature. It is evident that a strong temperature dependence of the absorption occurs only at longer wavelengths. The shorter the wavelength, the more the curves of all temperatures converge to one uniform value of $\kappa$. Generally, the mass absorption coefficient decreases with increasing wavelength and with decreasing temperature. The appearance of our curves is in good agreement with recent works by Boudet et al. (2005) and Coupeaud et al. (2011) [8]. It has to be mentioned that the absolute $\kappa$-values of the latter are in general higher than ours, which may be due to the fact, that their sample material was not a glass, but a sol-gel product. The data from Boudet et al. fits ours almost perfectly, except for the longest wavelengths where our measurements deliver slightly lower values.

The iron containing glasses show similar behavior to $\mathrm{MgSiO}_{3}$, only the absolute values of $\kappa$ are changing according to the incorporated iron fraction. Since the temperature dependence is significant at longer wavelengths, we will focus in the following on the spectral region $\lambda>0.5 \mathrm{~mm}$. Comparing our measurements with regard to the iron content, we find that $\mathrm{MgSiO}_{3}$, for some reason, is the best absorbing material. The presence of iron seems to lower the absorptivity in general, but apparently without following a constant trend. Room temperature mass absorption coefficients of samples with various iron contents are compared in Figure 2. The spectra are obtained only from measurements of $400 \mathrm{mg}$ pellets and the plotted values at wavelengths close to $1 \mathrm{~mm}$ may thus deviate from those of $2,4 \mathrm{~g}$ pellets, which are expected to provide more accurate values. Most likely, the actual curves would be located slightly lower than the ones shown. We have to apply the depicted curves until more experimental data of high mass pellets, especially for Fe-rich glasses, are available. However, the plot is totally suitable for demonstrating the basic behavior. Starting from $\mathrm{MgSiO}_{3}, \kappa$ decreases with increasing iron content up to the composition $\mathrm{Mg}_{0.7} \mathrm{Fe}_{0.3} \mathrm{SiO}_{3}$,

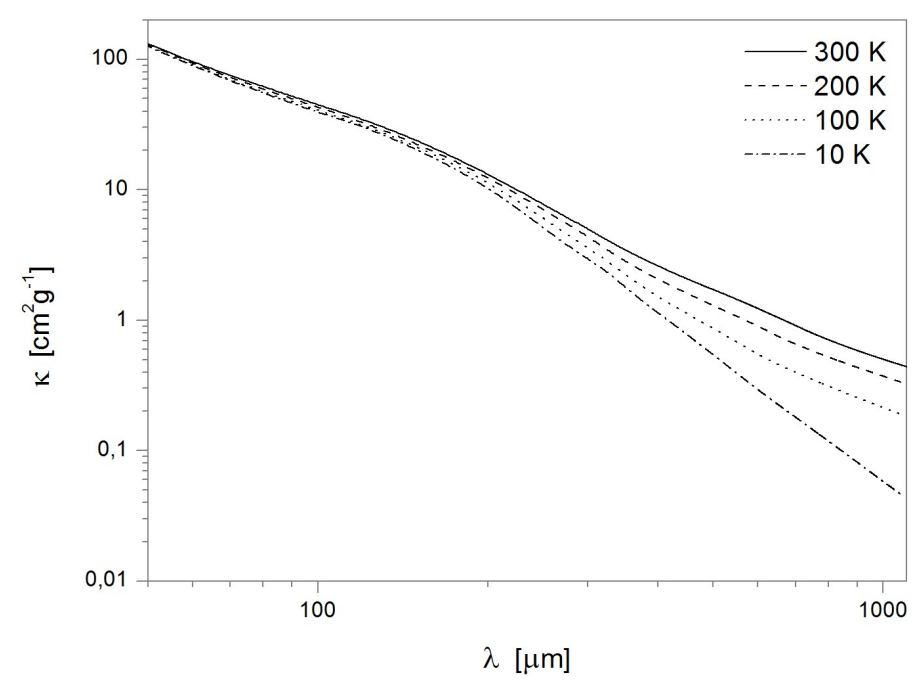

Fig. 1: Mass absorption coefficient for amorphous $\mathrm{MgSiO}_{3}$ at different temperatures 


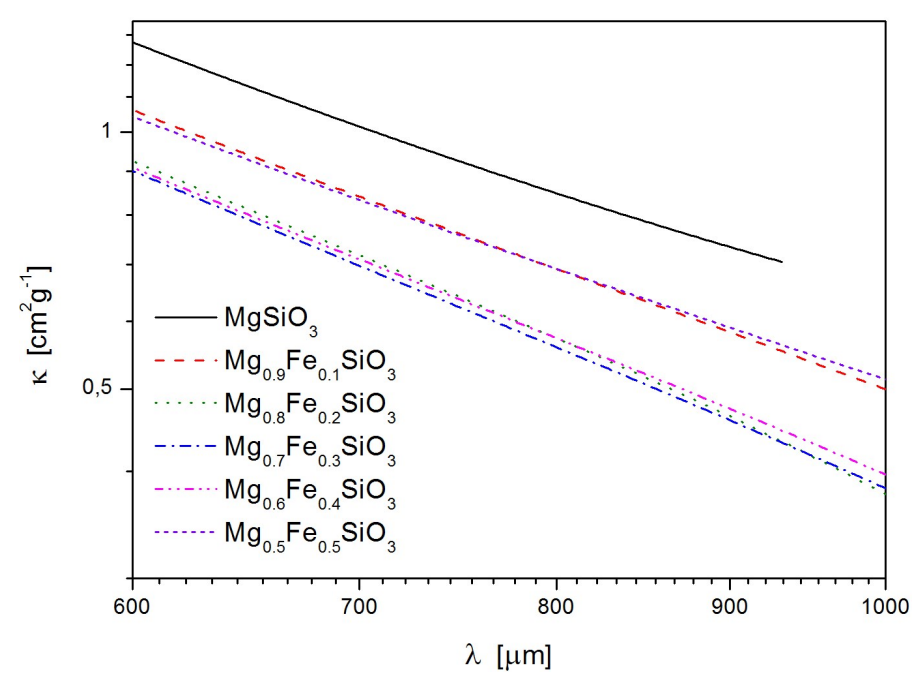

Fig. 2: $\kappa$-values at $300 \mathrm{~K}$ of amorphous pyroxene-like silicates with variable $\mathrm{Mg} / \mathrm{Fe}$-ratio $\left(\mathrm{Mg}_{x} \mathrm{Fe}_{1-x} \mathrm{SiO}_{3}\right.$; solid black: $x=1$, dashed red: $x=0.9$, dotted green: $x=0.8$, dash-dotted blue: $x=0.7$, dash-double-dotted magenta: $x=0.6$, short-dashed purple: $x=0.5$ )

which is the weakest absorber of our sample set. By incorporating still more iron in the amorphous network, the absorptivity rises again. We do not believe that this behavior is an intrinsic property of the amorphous silicates. Rather, it is caused either by $\mathrm{Fe}^{3+}$-induced disruptions of the network or by nanoscale iron inclusions like magnetite. Both alternatives are conceivable because the quenching process of our melts cannot be conducted under defined atmospheric conditions. However, detailed investigations on the microstructure of the glasses are necessary to understand the mechanisms governing the observed absorption. We plan to perform several structure elucidating measurements in the near future. The corresponding analytical methods are presented in the last section of this article.

\section{Future work}

We will continue and complete our measurements at the far infrared range and expand them, especially by reckoning glasses with olivine-like stoichiometry. It is also planned to expand the examined wavelength range towards longer wavelengths. For that purpose, a total power microwave spectrometer, equipped with a closed cycle LHe-cryostat, has already been designed and built (Cologne). First test of the setup have been carried out and went successfully. It is intended to take spectra up to a wavelength of $4 \mathrm{~mm}$.

Furthermore, it will be crucial to conduct a series of microstructural investigations on the glasses. We will apply Mössbauer spectroscopy to determine the ratio of divalent to trivalent iron in our samples, which may have major impact on the absorption properties. By now, one measurement of $\mathrm{Mg}_{0.7} \mathrm{Fe}_{0.3} \mathrm{SiO}_{3}$ has been carried out and revealed a ratio $\mathrm{Fe}^{2+} / \mathrm{Fe}^{3+}$ of almost exactly 1 . Of course we will have to obtain values for all our samples, which will be time-consuming because of the required integration time of about one week per measurement. Parallel to this we will apply Raman spectroscopy and high resolution transmission electron microscopy to determine the ratio 
of bridging to non-bridging oxygen and to check our samples for inclusions and possible mediumrange ordered areas, respectively. If necessary, we will expand the structural investigations to other methods, like NMR spectroscopy, in order to gain a clearer understanding of the possible processes governing the observed optical properties.

\section{Acknowledgements}

This work is part of the priority program 1573 "The Physics of the Interstellar Medium" of the DFG (Deutsche Forschungsgemeinschaft). We acknowledge the DFG for financial support.

\section{References}

[1] R.A. Bernstein, W.L. Freedman, and B.F. Madore, The first detections of the extragalactic background light at 3000, 5500, and 8000 angstrom. III. Cosmological implications, ApJ 571 (2002) 107

[2] N.J. Woolf and E.P. Ney, Circumstellar infrared emission from cool stars, ApJ 155 (1969) L181

[3] N.I. Agladze, A.J. Sievers, S.A. Jones, J.M. Burlitch, and V.W. Beckwith, Laboratory results on millimeter-wave absorption in silicate grain materials at cryogenic temperatures, ApJ 462 (1996) 1026

[4] N. Boudet, H. Mutschke, C. Nayral, C. Jaeger, J.-P. Bernard, T. Henning, and C. Meny Temperature dependence of the submillimeter absorption coefficient of amorphous silicate grains, ApJ 633 (2005) 272

[5] V. Mennella, J.R. Brucato, L. Colangeli, P. Palumbo, A. Rotundi, and E. Bussoletti Temperature dependence of the absorption coefficient of cosmic analog grains in the wavelength range 20 microns to 2 millimeters, ApJ 496 (1998) 1058

[6] J. Dorschner, B. Begemann, T. Henning, C. Jaeger, and H. Mutschke Steps toward interstellar silicate mineralogy II. Study of Mg-Fe-silicate glasses of variable composition, A\&A 300 (1995) 503

[7] T. Henning, B. Michel, and R. Stognienko Dust opacities in dense regions, Planet. Space Sci. 43 (1995) 1333

[8] A. Coupeaud, K. Demyk, C. Meny, C. Nayral, F. Delpech, H. Leroux, C. Depecker, G. Creff, J.-B. Brubach, and P. Roy Low-temperature FIR and submillimetre mass absorption coefficient of interstellar dust analogues, A\&A 535 (2011) A124 